\title{
Palestina versus Estados Unidos ante la Corte Internacional de Justicia*
}

\author{
Palestine vs. the United States before \\ the International Court of Justice*
}

\section{Palestina versus Estados Unidos ante a Corte Internacional de Justiça*}

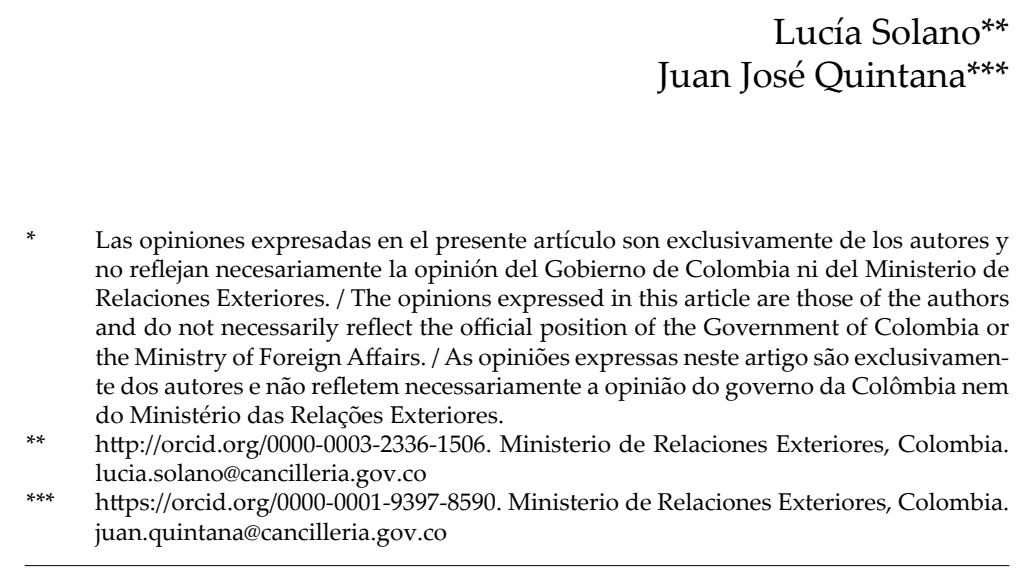

Recibido: 15/04/2020. Envío a pares: 21/04/2020 Aprobado por pares: 09/12/2020. Aceptado: 09/12/2020

DOI: $10.5294 /$ dika.2020.29.2.2

Para citar este artículo / To reference this article / Para citar este artigo Lucía Solano y Juan José Quintana, "Palestina versus Estados Unidos ante la Corte Internacional de Justicia”, en Díkaion 29, 2 (2020), 352-378. DOI: https://doi.org/10.5294/dika.2020.29.2.2 


\section{Resumen}

En el presente artículo se analiza la demanda interpuesta por Palestina contra Estados Unidos ante la Corte Internacional de Justicia, por las presuntas violaciones por parte de este último de la Convención de Viena sobre Relaciones Diplomáticas. La demanda surgió tras el anuncio del presidente estadounidense de trasladar la embajada norteamericana en Israel, de Tel Aviv a Jerusalén. El caso tiene importantes repercusiones de orden diplomático, pero además genera diversas preguntas jurídicas relacionadas con la cuestión del acceso a la Corte y, al respecto, con la condición o no de Estado de Palestina; con la competencia de esta y, en ese sentido, con la oponibilidad de las adhesiones de Palestina a la Convención y a su Protocolo Facultativo y, finalmente, con la admisibilidad de la demanda. En el escrito se analizará si Palestina podía iniciar un caso ante la Corte, si la Secretaría de la Corte debió aceptar que el caso fuera tramitado y si, de manera general, la Corte posee competencia para adjudicar el caso. El análisis de estos aspectos se hace con fundamento en las disposiciones del Estatuto y del Reglamento de la Corte, así como en la abundante jurisprudencia de esta en materia procesal.

\section{Palabras clave}

Corte Internacional de Justicia; asuntos contenciosos; acceso; competencia; admisibilidad; Estado; oponibilidad; excepciones preliminares. 


\section{Abstract}

This article discusses Palestine's lawsuit against the United States before the International Court of Justice for its alleged violations of the Vienna Convention on Diplomatic Relations. The lawsuit was brought after the US president's announcement to move the embassy in Israel from Tel Aviv to Jerusalem. The case has critical diplomatic repercussions but raises various legal questions related to Palestine's access to the Court with or without its capacity as a State, the Court's jurisdiction, the enforceability of Palestine's adherence to the Convention and its Optional Protocol, and the admissibility of the lawsuit. The paper discusses whether Palestine could initiate a case before the Court, whether the secretariat of the Court should have accepted the case, and whether the Court has jurisdiction to rule on it. This analysis is based on the provisions in the Court's bylaws, regulations, and extensive case law regarding procedural matters.

\section{Keywords}

International Court of Justice; contentious matters; access; competence; admissibility; State; enforceability; preliminary objections. 


\section{Resumo}

Neste artigo, é analisada a demanda interposta pela Palestina contra os Estados Unidos ante a Corte Internacional de Justiça, pelas supostas violações deste último da Convenção de Viena sobre Relações Diplomáticas. A demanda surgiu após o anúncio do presidente estadunidense de transferir a embaixada estadunidense em Israel de Tel Aviv a Jerusalém. O caso tem importantes repercussões de ordem diplomática, mas, além disso, gera diversas perguntas jurídicas relacionadas com a questão do acesso à Corte e, a respeito disso, com a condição ou não de Estado da Palestina; com a competência desta e, nesse sentido, com a oponibilidade das adesões da Palestina à Convenção e a seu Protocolo Facultativo; finalmente, com a admissibilidade da demanda. No texto, é analisado se a Palestina podia iniciar um caso ante a Corte, se a Secretaria da Corte deveu aceitar que o caso fosse tratado e se, de maneira geral, a Corte possui competência para adjudicar o caso. A análise desses aspectos é feita com base nas disposições do Estatuto e do Regulamento da Corte, bem como na abundante jurisprudência desta em matéria processual.

\section{Palavras-chave}

Corte Internacional de Justiça; assuntos litigiosos; acesso; competência; admissibilidade; Estado; oponibilidade; exceções preliminares. 
Sumario: Introducción. 1. La demanda de Palestina y la reacción de Estados Unidos. 2. La cuestión del acceso a la Corte Internacional de Justicia. 3. La cuestión de la competencia de la Corte. 4. Algunos aspectos de la Providencia del 15 de noviembre de 2018. 5. Consideraciones finales. Bibliografía.

\section{Introducción}

El 28 de septiembre de 2018, Palestina presentó una demanda en contra de Estados Unidos de América ante la Corte Internacional de Justicia (CIJ o la Corte). La demanda hace referencia a una controversia sobre presuntas violaciones por parte de Estados Unidos a la Convención de Viena sobre Relaciones Diplomáticas de 1961 (en adelante la Convención). ${ }^{1}$

Haciendo abstracción de las enormes repercusiones de índole diplomática que tiene una acción como esta, el caso genera diversas preguntas de orden legal $\mathrm{y}$, cualquiera que sea su resultado, tendrá consecuencias importantes para el derecho internacional y para el procedimiento que se sigue en asuntos contenciosos ante la CIJ.

Es importante recordar que el día 6 de diciembre de 2017 el presidente de Estados Unidos reconoció a Jerusalén como capital del Estado de Israel y anunció la relocalización de la embajada estadounidense de Tel Aviv a Jerusalén. Posteriormente, el 14 de mayo de 2018, la nueva embajada fue inaugurada en Jerusalén.

Adicionalmente, vale la pena destacar que la estatalidad de Palestina es una cuestión que genera profundos debates en el escenario internacional. A la fecha, 138 países $^{2}$ reconocen a Palestina como Estado, el último de los cuales fue Colombia, mediante un reconocimiento que efectuó el pasado 3 de agosto de 2018.

Este hecho es políticamente significativo, pero además y especialmente para efectos de la presente discusión, jurídicamente muy relevante con miras a establecer si Palestina tiene la capacidad legal para iniciar un caso contencioso contra otro Estado ante la CIJ, es decir, si posee acceso a esta. Únicamente si la respuesta a esta pregunta es afirmativa surge la cuestión subsidiaria de si a la luz de los instrumentos citados la CIJ posee competencia sobre la controversia llevada ante ella por Palestina.

1 El texto de la demanda se puede consultar en el sitio web de la Corte (https://www.icj-cij.org/en/case/176/ institution-proceedings).

2 Asimismo, las siguientes once organizaciones, cuerpos y tribunales han aceptado a Palestina como miembro: 1) Confederación Sindical Internacional, 2) Organización para la Cooperación Islámica, 3) Liga Árabe, 4) Comité Olímpico Internacional, 5) Federación Internacional de Fútbol Asociado (FIFA), 6) Organización Mundial del Turismo (como Observador), 7) Organización Mundial de la Salud (como Observador), 8) Media Luna Roja, 9) Organización de las Naciones Unidas para la Educación, la Ciencia y la Cultura (Unesco), 10) Corte Penal Internacional (CPI), y 11) Organización Internacional de Policía Criminal (Interpol). 
Así las cosas, surgen varias preguntas concretas: ¿podía Palestina iniciar un caso ante la CIJ? ¿Debió la Secretaría de la CIJ aceptar que el caso fuera tramitado? De manera general, ¿posee la CIJ competencia para adjudicar entre Palestina y Estados Unidos?

Estas cuestiones se analizarán en los apartes siguientes.

\title{
1. La demanda de Palestina y la reacción de Estados Unidos
}

A través de su demanda, de fecha 28 de septiembre de 2018, Palestina le solicita a la CIJ que resuelva una controversia surgida entre ella y Estados Unidos en relación con la relocalización de la embajada estadounidense en Israel a la ciudad santa de Jerusalén.

Palestina argumenta que de una simple lectura de la Convención se desprende fácilmente que la misión diplomática del Estado acreditante debe establecerse en el territorio del Estado receptor. En consecuencia, para Palestina, a la luz del estatus especial de Jerusalén, "la reubicación de la Embajada de los Estados Unidos en Israel en [...] Jerusalén constituye una violación de la Convención de Viena". ${ }^{3}$

Sobre el "estatus especial" de la ciudad de Jerusalén en el derecho internacional, Palestina añade que esto obedece a sus "dimensiones espirituales, religiosas y culturales únicas", y que por tanto las Naciones Unidas ha adoptado diversas resoluciones que buscan protegerla. Dice Palestina:

\begin{abstract}
Desde el 29 de noviembre de 1947, la Asamblea General de las Naciones Unidas adoptó un Plan de Partición a través de su Resolución 181 (II), Futuro Gobierno de Palestina estableciendo "Estados árabes y judíos independientes y el Régimen Especial Internacional para la ciudad de Jerusalén" en Palestina. Adicionalmente especificó que: "la ciudad de Jerusalén se establecerá como un cuerpo separado bajo un régimen internacional especial".
\end{abstract}

Como fundamento de la competencia de la CIJ, Palestina invocó el artículo $1^{\circ}$ del Protocolo Facultativo sobre la Jurisdicción Obligatoria para la Solución de Controversias de la Convención de Viena sobre Relaciones Diplomáticas de

3 Sin embargo, es interesante recalcar que en la demanda no se invoca ninguna norma específica de la Convención. Por curioso que pueda parecer, en ninguna de las disposiciones de la Convención de Viena se establece en forma explícita que las misiones diplomáticas deben estar situadas en la localidad donde el Estado receptor tiene su capital o la sede de gobierno.

4 Demanda de Palestina, p. 2 (la traducción es de los autores, al igual que en los restantes extractos de jurisprudencia y doctrina). 
1961 (Protocolo Facultativo), instrumentos de los cuales tanto Palestina ${ }^{5}$ como Estados Unidos ${ }^{6}$ tendrían la condición de Estado parte. Adicionalmente, el 4 de julio de 2018, Palestina depositó una declaración de reconocimiento de la competencia obligatoria de la CIJ para la solución de todas las controversias que ya hayan surgido o llegasen a surgir en el futuro en relación con los artículos I y II del Protocolo Facultativo.

Por ende, Palestina solicita a la CIJ que "declare que la reubicación, a la Ciudad Santa de Jerusalén, de la embajada de los Estados Unidos en Israel viola la Convención de Viena"; además, pide a la CIJ que "ordene a los Estados Unidos de América que retire la misión diplomática de la Ciudad Santa de Jerusalén y respete sus obligaciones internacionales en virtud de la Convención de Viena"; finalmente, le solicita que decrete que Estados Unidos debe "tomar todas las medidas necesarias para cumplir con sus obligaciones y que ofrezca seguridades y garantías de no repetición de su conducta ilegal".

El 2 de noviembre de 2018, al ser notificado de la demanda, Estados Unidos envió a la CIJ una comunicación en la cual le informa que, en su opinión, es manifiesto que ella carece de competencia para conocer de la demanda de Palestina y, por tanto, le solicita remover el caso de su Lista General. Además, ese Gobierno anunció que se abstendría de designar agente y que no asistiría a una reunión del presidente de la CIJ con los agentes convocada para poco después.

De lo anterior se infiere que, por ahora, Estados Unidos ha decidido no comparecer en este proceso. En una providencia adoptada el 15 de noviembre de 2018, la CIJ decidió que la primera ronda de alegatos escritos en este caso se dedique a las cuestiones de competencia de la CIJ y admisibilidad de la demanda que han sido planteadas por Estados Unidos y fijó plazos para el depósito de dichos alegatos. En el acápite final del presente artículo se hacen unas consideraciones respecto de esta decisión. ${ }^{7}$

A la luz de los presupuestos fácticos precedentes, a continuación se analizará si la demanda interpuesta por Palestina puede ser estudiada por la CIJ.

Palestina adhirió a la Convención el 2 de abril de 2014 y al Protocolo Facultativo el 22 de marzo de 2018. Estados Unidos es parte de ambos instrumentos desde el 13 de noviembre de 1972.

Está muy claro que la Corte ha decidido seguir adelante con el procedimiento en este caso, el cual fue ingresado en la Lista General. Nótese que i) la Corte se negó a acceder a la solicitud de Estados Unidos de retirar el caso de su Lista General, o sea que declinó aplicar el concepto de "manifest lack of jurisdiction", y ii) aunque la actitud de Estados Unidos hasta ahora apunta claramente a que se configure en este caso una situación de no comparecencia, por el momento la Corte no ha sentido la necesidad de invocar la regla del artículo 53 del Estatuto, que contempla las consecuencias jurídicas que se desprenden de dicha situación. Esto podría resultar necesario más adelante, en el evento de que la Corte encuentre que posee competencia y decida continuar con el proceso. Como es evidente, la situación procesal también podría modificarse en sentido contrario, por ejemplo, si en la eventualidad anotada Estados Unidos decide cambiar de curso y comparecer durante la fase de fondo. 


\section{La cuestión del acceso a la Corte Internacional de Justicia}

Acceso y competencia. En primer lugar, y en el marco de lo hasta ahora expuesto, es importante aclarar la diferencia que existe entre los conceptos de acceso y competencia.

En efecto, con miras a que la CIJ pueda asumir conocimiento de un caso contencioso que se le presente se deben cumplir dos requisitos acumulativos:

i. Que todos los Estados que participen en el proceso pertenezcan a aquellos grupos de Estados para los cuales está disponible la CIJ, vale decir que tengan acceso a esta.

ii. Que exista competencia de la CIJ sobre el caso.

Así, en tanto la CIJ solo puede conocer de un asunto si los Estados implicados han consentido en pasar a ser partes en actuaciones ante esta, aun si un Estado tiene la capacidad de comparecer ante este tribunal ello no implica automáticamente que la CIJ tenga competencia sobre ese Estado y sus actuaciones. En otras palabras, que un Estado pueda acceder a la CIJ (locus standi in judicio) no significa que tenga que presentarse ante esta o que dicho tribunal tenga inmediatamente competencia ratione personae sobre este. ${ }^{8}$

Sobre el particular, en el caso sobre Kosovo, ${ }^{9}$ la CIJ afirmó lo siguiente:

La Corte, bajo su Estatuto, no tiene competencia automática sobre controversias jurídicas entre Estados partes del Estatuto o entre otros Estados a quienes les está permitido el acceso a la Corte [...] la Corte puede, por tanto, ejercer competencia solo entre Estados partes de una controversia que no solo tengan acceso a la Corte, sino que además también hayan aceptado la competencia de la Corte, sea de forma general o para la controversia específica respectiva. (Énfasis agregado)

Se desprende de ello que, en línea con el principio de la autonomía de la voluntad, para que la CIJ posea competencia ratione personae $\mathrm{y}$, por ende, pueda entrar a conocer de un caso, debe primero analizar que concurra el acceso a esta $\mathrm{y}$, después, revisar si las partes que poseen dicho acceso aceptaron además su

8 Para un discusión general sobre el tema del acceso a la Corte, se recomienda ver: Juan José Quintana, Litigation at the International Court of Justice: Practice and Procedure, Leiden, Brill, 2015; Shabtai Rosenne, The Law and Practice of the International Court: 1920-2015, Dordrecht, Brill Nijhoff, 2006; Hugh Thirlway, The International Court of Justice, Oxford, Oxford University Press, 2016; Andreas Zimmermann, Christian Tomuschat y Karin Oellers-Frahm (eds.), The Statute of the International Court of Justice: A Commentary, London, Oxford University Press, 2006.

9 Corte Internacional de Justicia, “Caso relativo a la legalidad del uso de la fuerza (Serbia y Montenegro v. Bélgica), Medidas Provisionales", Providencia de 2 de junio de 1999, ICJ Reports 1999, p. 266, pár. 22. 
competencia. En efecto, en el mismo caso en comento entre Serbia-Montenegro y Francia, ${ }^{10}$ la CIJ señaló:

La Corte puede ejercer su función judicial solo con respecto a aquellos Estados que tienen acceso a ella bajo el artículo 35 del Estatuto. Y solo aquellos Estados que tienen acceso a la Corte pueden conferirle jurisdicción para el efecto.

En opinión de la Corte, recae sobre ella examinar, en primer lugar, la cuestión de si un Demandante cumple con los requisitos establecidos en los artículos 34 y 35 del Estatuto y si la Corte está, por tanto, abierta para este. Solo si la respuesta a esa cuestión es afirmativa podrá la Corte entrar a resolver los asuntos relativos a las condiciones establecidas en el Artículo 36 del Estatuto de la Corte.

Por tanto, si una entidad no tiene acceso a la CIJ al no estar cubierta por ninguna de las categorías descritas en el acápite precedente, no puede manifestar su consentimiento a la competencia de esta de una forma válida y, por ende, no tendría la posibilidad de dar origen a la competencia de ese tribunal. ${ }^{11} \mathrm{En}$ términos sencillos, la competencia de la CIJ no puede activarse si el respectivo demandante no tenía "derecho a comparecer" ante esta.

El asunto del acceso, entonces, debe ser analizado siempre y en primer lugar por la CIJ, antes del análisis sobre competencia, e incluso si las partes no le han solicitado expresamente que lo estudie; es decir, el análisis debe hacerse de oficio. Así lo reiteró la CIJ en el caso del Genocidio, en la etapa de fondo, ${ }^{12}$ cuando expresó que:

Por ende, si la Corte considera que, para un caso concreto, las condiciones relativas a la capacidad de las partes para comparecer frente a ella no están satisfechas, aunque las condiciones de su competencia ratione materiae sí lo estén, deberá, incluso si el asunto no ha sido planteado por las partes, encontrar que las primeras condiciones no se cumplieron, y concluir, por esa razón, que no tiene competencia para decidir sobre el fondo.

Para el caso interpuesto por Palestina en contra de Estados Unidos, por consiguiente, incluso si la CIJ tuviese competencia en virtud del artículo $1^{\circ}$ del Protocolo Facultativo, como lo sostiene Palestina, primero debe establecer si Palestina tenía acceso a ella y, como se explica en el acápite subsiguiente, la respuesta a esa pregunta podría ser negativa.

10 Corte Internacional de Justicia, “Caso relativo a la Legalidad del Uso de la Fuerza (Serbia y Montenegro v. Francia), Medidas Provisionales", Sentencia de 15 de diciembre de 2004, ICJ Reports 2004, p. 594, pár. 45.

11 Idem.

12 Corte Internacional de Justicia, “Caso relativo a la aplicación de la Convención sobre la Prevención y el Castigo del Delito de Genocidio (Bosnia Herzegovina v. Serbia y Montenegro), Fondo", Sentencia de 26 de febrero de 2007, ICJ Reports 2007, p. 94, pár. 122. Para un análisis sobre los casos de Yugoslavia ante la CIJ y la cuestión del acceso, se recomienda ver, Shabtai Rosenne, "Capacity to litigate in the International Court of Justice: Reflections on Yugoslavia in the Court", en British Yearbook of International Law 80 (1) (2009), pp. 217-243. 
Reglas sobre acceso a la CIJ y la situación de Palestina. De conformidad con el artículo 34 del Estatuto de la Corte Internacional de Justicia, ${ }^{13}$ solo los Estados podrán ser partes en asuntos contenciosos ante la $\mathrm{CIJ}$ y, por tanto, presentarle casos.

Sin embargo, a la luz de la Carta de las Naciones Unidas (en adelante, la Carta) y el Estatuto, no todos los Estados pueden comparecer frente a la CIJ, sino que solo tienen acceso a ella aquellos que pertenecen a algunas de las siguientes tres categorías:

1. Los Estados miembros de las Naciones Unidas que al ratificar la Carta aceptaron sus obligaciones $\mathrm{y}$, por tanto, automáticamente pasaron a ser partes en el Estatuto de la CIJ, que forma parte de la Carta, según lo previsto en el artículo 35, párrafo 1 del Estatuto.

2. Los Estados que hayan pasado a ser parte en el Estatuto de la CIJ, aun sin ser miembros de las Naciones Unidas, en virtud del artículo 93, párrafo 2 de la Carta de las Naciones Unidas.

3. Los Estados que, aunque no sean miembros de las Naciones Unidas ni parte en el Estatuto de la CIJ, hayan depositado en la Secretaría una declaración que reúna los requisitos establecidos por el Consejo de Seguridad en cuya virtud acepten la jurisdicción de la CIJ y se comprometan a dar cumplimiento de buena fe a sus decisiones, a la luz del artículo 35, párrafo 2 del Estatuto.

En cuanto al primer grupo de Estados, i.e., aquellos que tienen acceso a la CIJ por ser miembros de las Naciones Unidas y partes en el Estatuto de la Corte, no cabe duda de que Palestina no ha sido admitida como miembro de esta organización, sino simplemente como observador, a través de la Resolución de la Asamblea General A/Res/67/19 de 4 de diciembre de 2012, que dispone lo siguiente:

Decide conceder a Palestina la condición de Estado observador no miembro en las Naciones Unidas, sin perjuicio de los derechos adquiridos, las prerrogativas y la función de la Organización de Liberación de Palestina en las Naciones Unidas como representante del pueblo palestino, de conformidad con las resoluciones y la práctica pertinentes. (Énfasis agregado)

Esta resolución tuvo, adicionalmente, mucha oposición de parte de Estados Unidos y del Estado de Israel, entre otras naciones.

Dada la claridad de esta resolución en cuanto al estatus de Palestina frente a las Naciones Unidas parecería evidente que, al no tener todavía la condición

13 El artículo 34 dispone lo siguiente:

"Artículo 34. 1. Solo los Estados podrán ser partes en casos ante la Corte". 
de miembro de la Organización, ni parte en el Estatuto de la Corte, no estaría en el primer grupo de Estados que tienen acceso a la CIJ. ${ }^{14}$

El segundo grupo de Estados, es decir, aquellos que pasaron a ser parte en el Estatuto de la Corte aun sin ser miembros de las Naciones Unidas, según las condiciones establecidas por la Asamblea General bajo recomendación del Consejo de Seguridad (art. 93, párr. 2 de la Carta de las Naciones Unidas), no es aplicable al caso concreto, toda vez que Palestina no ha iniciado siquiera los procedimientos exigidos para el efecto. ${ }^{15}$

Lo anterior tiene sentido en la medida que Palestina ha buscado, hasta ahora sin éxito, que se le tenga como Estado miembro de las Naciones Unidas (lo cual implica ratificar la Carta de la ONU) y, por tanto, ha decidido no iniciar un procedimiento para ratificar únicamente el Estatuto de la Corte, es decir, para ser parte del segundo grupo. Hacer eso no solamente iría en contra de su aspiración a ser miembro pleno, sino que, adicionalmente, tendría pocas probabilidades de éxito ya que el procedimiento debe iniciarse por recomendación del Consejo de Seguridad y no pareciera factible que esto sucediera en las circunstancias actuales dada la composición del Consejo.

Finalmente, hay que analizar si Palestina pertenece al tercer grupo, es decir, aquellos Estados que, sin ser miembros de las Naciones Unidas ni parte en el Estatuto, han depositado en la Secretaría de la CIJ una declaración que reúne los requisitos establecidos por el Consejo de Seguridad en la Resolución 9, adoptada el 15 de octubre de 1946, la cual desarrolla lo previsto en el artículo 35, párrafo 2 del Estatuto.

Según los términos de esta resolución del Consejo, quien quiera aceptar como obligatoria la competencia de la CIJ en relación con cualquier otro Estado que acepte la misma obligación, puede depositar una declaración unilateral en la que reconozca esa competencia y se comprometa a cumplir de buena fe las decisiones de la CIJ y a aceptar todas las obligaciones que surgen para un miembro de la Organización según el artículo 94 de la Carta.

14 Una circunstancia similar ocurre en el caso de ausencia de reconocimiento de un Estado. Por ejemplo, en el asunto sobre la Convención contra el Genocidio (Bosnia-Herzegovina v. Serbia y Montenegro), Serbia y Montenegro presentó excepciones preliminares argumentando que el demandante y el demandado no se reconocían mutuamente como Estados y que, por tanto, la Corte no tenía competencia para resolver el asunto. Sin embargo, la Corte no tuvo que resolver este asunto toda vez que cuando emitió su decisión sobre las excepciones preliminares notó que esta situación ya no era relevante porque los Estados habían firmado el acuerdo de Dayton-París, que conllevó el reconocimiento recíproco de todos los Estados que formaban parte de la antigua Yugoslavia (Corte Internacional de Justicia, "Caso relativo a la Aplicación de la Convención sobre la Prevención y el Castigo del Delito de Genocidio [Bosnia Herzegovina v. Serbia y Montenegro], Excepciones Preliminares", Sentencia de 11 de julio de 1996, ICJ Reports 1996, pp. 612-613, pár. 25).

15 Hoy no existe ningún Estado en esta categoría. Para aquellos que solicitaron este estatus en el pasado, los requisitos, según aparecen descritos en la Resolución No. 91 del 11 de diciembre de 1946, en la que se analizó la solicitud de Suiza, fueron: a) una aceptación general del Estatuto; b) un compromiso expreso frente a las obligaciones derivadas de la condición de miembro según el artículo 94 de la Carta, y c) un compromiso de contribuir a los gastos ocasionados por la Corte en una escala a ser fijada por la Asamblea General. 
En teoría, esta resolución permitiría el recurso a la CIJ a una entidad cuyo reconocimiento como Estado esté en discusión, con el simple hecho de depositar la declaración en mención. Sin embargo, la resolución es muy precisa al precisar que solo los Estados están autorizados a depositar una declaración de esta naturaleza. La misma resolución aclara, en su numeral 5, que las dudas sobre la "validez o los efectos de una declaración hecha con arreglo a lo establecido en esta resolución serán decididas por la Corte".

Palestina efectivamente depositó ante la Secretaría de la CIJ, el 4 de julio de 2018, una declaración en la que reconocía la competencia de la Corte para que resolviese todas las controversias que puedan surgir o hubiesen surgido y que estuvieran cubiertas por los artículos I y II del Protocolo Facultativo, manifestando para el efecto que lo hace en concordancia con la Resolución 9 del Consejo y el artículo 35 párrafo 2 del Estatuto.

En consecuencia, el demandante se cataloga a sí mismo como perteneciente al tercer grupo de Estados que puede tener acceso a la CIJ. Sin embargo, como es de conocimiento general, existe amplio debate en el escenario internacional sobre si el asunto de la condición de Estado de Palestina bajo el derecho internacional ha sido resuelto definitivamente, y esa condición de Estado es requisito necesario para acceder a la $\mathrm{CIJ}^{16} \mathrm{y}$, por ende, para que esta se declare competente para asumir conocimiento del caso concreto. ${ }^{17}$

Ciertamente, la Resolución A/Res/67/19 de 4 de diciembre de 2012, por la que la Asamblea General de la ONU decide conceder a Palestina la condición de Estado observador no miembro en ese órgano, reafirma el derecho de Palestina a tener una existencia independiente como Estado y registra que muchos Estados miembros de las Naciones Unidas lo han reconocido como tal. Sin embargo, no cabe duda de que esta resolución por sí misma no resuelve, ni pretendía resolver, el asunto de la condición de Estado de Palestina.

Adicionalmente, las condiciones normativas para obtener la condición de Estado en derecho internacional no admiten mayor discusión, ya que se considera generalmente aceptado que estas condiciones fueron formuladas en

16 Se han escrito varios artículos y entradas de blog sobre la condición de Estado de Palestina y su capacidad para presentar un caso ante la Corte, los cuales se recomienda consultar: Charles F. Whitman, "Palestine's statehood and ability to litigate in the International Court of Justice", en California Western International Law Journal 44 (1) (2013), art. 4; Jure Vidmar, "Palestine v United States: Why the ICJ does not need to decide whether Palestine is a state", en European Journal of International Law (EJIL: Talk!) (2018), en https://www.ejiltalk.org/ palestine-v-united-states-why-the-icj-does-not-need-to-decide-whether-palestine-is-a-state/. En este, el autor alega que no es necesario que la Corte defina si Palestina es un Estado o no para efectos de decidir si la Corte debe conocer de la demanda interpuesta contra Estados Unidos.

17 Algunos autores han expresado críticas frente al hecho de que se requiera ser Estado para poder acceder a la Corte en un caso contencioso. Al respecto, ver por ejemplo: Pierre-Marie Dupuy, "Artículo 34", en Andreas Zimmermann, Christian Tomuschat y Karin Oellers-Frahm, The Statute of the International Court of Justice, London, Oxford University Press, 2012, p. 585. 
forma precisa en la Convención de Montevideo sobre Derechos y Deberes de los Estados de 1933, la cual dispone en su artículo 1 lo siguiente:

Artículo 1.

El Estado como persona de Derecho Internacional debe reunir los siguientes requisitos:

I. Población permanente.

II. Territorio determinado.

III. Gobierno.

IV. Capacidad de entrar en relaciones con los demás Estados.

Estos requisitos son independientes del reconocimiento que los Estados emitan en escenarios como la Asamblea General de las Naciones Unidas, bilateralmente, o en otros foros, reconocimiento que, aunque puede basarse en los requisitos antes transcritos de la Convención de Montevideo, suele tener en cuenta motivaciones de orden político y otro tipo de consideraciones similares, tal y como ocurrió en el caso de la Resolución 67/19.

En consecuencia, definir si Palestina puede considerarse un Estado para los propósitos del caso en comento es un asunto de orden jurídico que en principio corresponderá definir de forma independiente a la CIJ, en virtud de las reglas sobre interpretación de tratados y aplicando las normas del derecho internacional general relativas a la condición de Estado. ${ }^{18}$

En ese sentido, el tribunal deberá empezar por establecer si, en efecto, Palestina podía legalmente depositar la declaración de aceptación de su competencia a la luz de los requisitos establecidos en la Resolución de $1946 .{ }^{19}$ Solo cuando decida si Palestina tiene acceso a la CIJ a la luz de la norma especial contenida en el artículo 35 de la Carta podrá esta, a su turno, analizar si tiene competencia sobre los asuntos que le fueron sometidos.

18 Un debate muy interesante acerca de la condición de Estado de Palestina se ha generado recientemente en sede de la CPI con ocasión de la determinación de si esa Corte tiene jurisdicción para decidir sobre la eventual comisión de delitos de su competencia por un Estado no parte en el Estatuto de Roma (Israel), en territorio que se alega ser de Palestina. Para los interesados en este tema se recomienda leer las observaciones y los escritos de amicus curiae presentados por diversos Estados, académicos, ONG y otro tipo de organizaciones a la Sala de Cuestiones Preliminares I de la CPI, para asistirle en la determinación del asunto jurisdiccional establecido en el párrafo 220 de la "Solicitud de la Fiscalía en virtud del artículo 19(3), para que la Corte decida sobre la competencia territorial en Palestina". Todos los documentos presentados se pueden encontrar en Corte Penal Internacional, "Court Records", en https://www.icc-cpi.int/Pages/crmrefined.aspx?situation $=\mathrm{ICC}-01 / 18 \#$ Default $=\% 7 \mathrm{~B} \% 22 \mathrm{k} \% 22 \% 3 \mathrm{~A} \% 22 \% 22 \% 7 \mathrm{D} \# 0454 \mathrm{~d} 5 \mathrm{ba}-9559-4 \mathrm{bcd}-9519$ $746 \mathrm{c} 1911 \mathrm{~b} 2 \mathrm{e} 5=\% 7 \mathrm{~B} \% 22 \mathrm{k} \% 22 \% 3 \mathrm{~A} \% 22 \% 22 \% 7 \mathrm{D}$

19 Por ejemplo, en el caso relativo a la Convención contra el Genocidio (Croacia v. Serbia y Montenegro), la Corte registró en términos expresos que ambas partes en el caso tenían la condición de "Estados" para los fines del artículo 34 del Estatuto (Corte Internacional de Justicia, “Caso relativo a la Aplicación de la Convención sobre la Prevención y el Castigo del Delito de Genocidio [Croacia v. Serbia], Excepciones Preliminares", Sentencia de 18 de noviembre de 2008, ICJ Reports 2008, p. 430, pár. 59). En forma análoga, en su temprano Dictamen sobre las condiciones para admisión en la $\mathrm{ONU}$, la Corte había resaltado que una condición sine qua non para ser admitido a esa organización es la de "ser un Estado" (Corte Internacional de Justicia, "Admisión a la ONU", Opinión Consultiva de 28 de mayo de 1948, ICJ Reports 1948, p. 62). 


\section{La cuestión de la competencia de la Corte}

Con miras a un análisis completo de la situación objeto de examen, se procede analizar si tanto Palestina como Estados Unidos pueden considerarse como parte de la Convención y de su Protocolo Facultativo sobre solución de controversias.

Al respecto, la Convención dispone lo siguiente en cuanto a la posibilidad de hacerse parte de esta:
Artículo 48
La presente Convención estará abierta a la firma de todos los Estados Miembros de las Naciones Unidas o de algún organismo especializado, así como de todo Estado Parte en el Estatuto de la Corte Internacional de Justicia y de cualquier otro Estado invitado por la Asamblea General de las Naciones Unidas a ser parte en la Convención, de la manera siguiente: hasta el 31 de octubre de 1961, en el Ministerio Federal de Rela- ciones Exteriores de Austria; y después, hasta el 31 de marzo de 1962, en la Sede de las Naciones Unidas en Nueva York. (Énfasis agregado).

En virtud de esta disposición, la Convención está abierta a entidades que no necesariamente son Estados miembros de las Naciones Unidas. Para el caso sub examine, como se indicó anteriormente, Palestina es miembro de varios organismos especializados de las Naciones Unidas y es en esta calidad que pudo hacerse parte de la Convención.

Por su parte, el artículo del Protocolo Facultativo a la Convención establece: "Artículo VII. El presente Protocolo quedará abierto a la adhesión de todos los Estados que puedan ser Partes en la Convención. Los instrumentos de adhesión se depositarán en poder del Secretario General de las Naciones Unidas".

En desarrollo de estas disposiciones, Palestina se hizo parte de la Convención el 2 de abril de 2014 y del Protocolo Facultativo el 22 de marzo de 2018.

No obstante, el 13 de mayo de 2014, a propósito de lo que llama una "presunta adhesión de Palestina a la Convención", Estados Unidos depositó una comunicación ante el Secretario General de las Naciones Unidas en la que declara que no se considera vinculado por esa Convención con respecto a Palestina y, asimismo, el $1^{\circ}$ de mayo de 2018 depositó una declaración adicional, en cuanto se conoció la adhesión de Palestina al Protocolo Facultativo, reiterando su posición. Finalmente, el 12 de octubre de 2018, el Gobierno de Estados Unidos notificó su denuncia del Protocolo Facultativo. ${ }^{20}$

20 La comunicación de Estados Unidos dice lo siguiente:

“... el Gobierno de los Estados Unidos de América [se refiere] al Protocolo Facultativo sobre la Jurisdicción Obligatoria para la Solución de Controversias de la Convención de Viena sobre Relaciones Diplomáticas, hecho 
A la luz de estas posiciones es cuestionable si, como cuestión propia del derecho de los tratados, se puede catalogar a Palestina como parte de la Convención y del Protocolo Facultativo vis-à-vis Estados Unidos.

Un primer factor que hay que considerar es que, si las adhesiones de Palestina a la Convención y a su Protocolo Facultativo son válidas, y más concretamente son oponibles frente a Estados Unidos, la denuncia del Protocolo hecha por este último carece de efectos frente al caso en comento. La razón para ello es que en materia de extinción de títulos de competencia la jurisprudencia de la CIJ ha sido constante en la reafirmación del llamado "principio Nottebohm" (llamado así por el caso en cuyo contexto fue formulado por primera vez, en los años cincuenta), el cual establece que, como la llamada "fecha crítica" para fines de jurisdicción es la del depósito de la demanda, ningún hecho ocurrido después de esa fecha y que afecte un título de competencia ya invocado ante la CIJ puede alterar la situación jurídica que existía en el momento del sometimiento del caso.

Los hechos del presente caso son muy claros: la demanda de Palestina fue presentada el 28 de septiembre de 2018, invocando como título exclusivo de competencia el Protocolo Facultativo, al cual Palestina había adherido desde el 22 de marzo del mismo año. ${ }^{21}$ La denuncia del Protocolo por Estados Unidos tuvo lugar el 12 de octubre de 2018 y, por tanto, el hecho de que ese país haya quedado desvinculado de ese tratado a partir de esa fecha no puede tener efecto alguno sobre la situación jurídica que existía entre Palestina y Estados Unidos en la fecha de la presentación de la demanda, esto es, el 28 de septiembre de 2018.

Sin embargo, lo que no está para nada claro es que la Convención de Viena y su Protocolo Facultativo sean tratados vigentes entre esos dos Estados. El derecho de los tratados no es sino una adaptación al plano internacional del derecho de los contratos en el derecho civil doméstico y, bajo ambos ordenamientos, un requisito fundamental, casi que ontológico, para la existencia de una relación contractual es que las partes tengan la voluntad de entrar en dicha relación. En otras palabras, así como en el derecho interno nadie puede ser obligado a vincularse por un contrato en contra de su voluntad, en el derecho internacional ningún Estado está obligado a entrar en relaciones convencionales con un Estado o entidad con la cual no quiera hacerlo.

en Viena el 18 de abril de 1961. Esta carta constituye una notificación por los Estados Unidos de América de que por medio de esta denuncia el precitado Protocolo. Como consecuencia de su denuncia, los Estados Unidos no seguirá reconociendo la competencia de la Corte Internacional de Justicia reflejada en ese Protocolo".

21 Corte Internacional de Justicia, "Nottebohm (Liechtenstein v. Guatemala)", Excepciones Preliminares, Sentencia de 18 de noviembre de 1953, ICJ Reports 1953, pp. 122-123. Una formulación clara del principio se puede encontrar en Corte Internacional de Justicia, "Caso relativo al derecho de paso sobre territorio indio (Portugal v. India)", Excepciones Preliminares, Sentencia de 26 de noviembre de 1957, ICJ Reports 1957, p. 142). 
En el momento mismo en que Estados Unidos fue notificado por el Secretario General de la ONU de que Palestina había presentado un instrumento de adhesión a la Convención y al Protocolo, ese Estado reaccionó y planteó en términos claros que Estados Unidos no aceptaba entrar en una relación convencional (a treaty relationship) con Palestina a la luz de ninguno de dichos instrumentos. Frente a una objeción tan terminante, es por lo menos dudoso que en la fecha de presentación de la demanda la Convención y su Protocolo Facultativo fueran tratados en vigor entre Palestina y Estados Unidos.

Ahora bien, podría ser posible que un Estado, aunque no sea miembro de las Naciones Unidas ni parte en el Estatuto de la Corte hubiera depositado en la Secretaría una declaración aceptando la jurisdicción de la CIJ para así incluirse dentro del tercer grupo de Estados que tienen acceso a este tribunal, pero esto tampoco parece ser el caso para Palestina como se explicó en los apartes anteriores.

Así las cosas, es difícil aceptar de manera general que la competencia de la CIJ se puede perfeccionar y quedar plenamente establecida únicamente con que el demandante cumpla la condición de ser parte de un tratado que le otorga competencia a la CIJ. La razón es que, si bien los términos de un tratado pueden permitir que se hagan parte del mismo entidades, organismos y sujetos que no necesariamente sean Estados, para poder activar la competencia de la Corte se requiere siempre tener la condición jurídica de Estado en los términos precisos del artículo 34 de su Estatuto. Como se dijo atrás, este es un punto preliminar que probablemente deberá resolver la CIJ cuando acometa la interpretación de la Declaración formulada por Palestina el 4 de julio de 2018.

\section{Algunos aspectos de la Providencia del 15 de noviembre de 2018}

Estructura. La Providencia es relativamente corta. Primero la CIJ recapitula los escasos desarrollos del procedimiento que se han presentado hasta ahora, entre ellos:

- La formulación de la "Declaración" de aceptación de competencia por Palestina el 4 de julio de 2018;

- la presentación y notificación de la demanda;

- la base de competencia aducida por el demandante (el Protocolo Facultativo de 1961);

- la designación de un agente por Palestina;

- la convocatoria de una reunión entre el presidente y representantes de las partes; 
- la comunicación recibida de parte del Gobierno de Estados Unidos, y

- la reunión celebrada el 5 de noviembre entre el presidente de la CIJ y los representantes de Palestina únicamente y la posición expresada por estos con respecto a aspectos del procedimiento.

Segundo, luego de unas breves consideraciones jurídicas, la CIJ toma dos decisiones que incorpora en la parte dispositiva, a saber: i) que los alegatos escritos o piezas del procedimiento deben abordar primero que todo la cuestión de la competencia de la CIJ y la admisibilidad de la demanda; y ii) que Palestina como demandante debía ser el primero, presentando una Memoria el 15 de mayo de 2019. Estados Unidos, como demandado, debía contestar presentando una Contramemoria el 15 de noviembre de 2019.

En cuanto a las consideraciones jurídicas que sirven de fundamento a estas decisiones, que son las dos que se explican a continuación, estas ameritan unos comentarios puntuales:

- Primer considerando: que, de conformidad con el artículo 79, párrafo 2 de su Reglamento, en las circunstancias del caso la CIJ considera que debe primero que todo resolver las cuestiones de la competencia de la Corte y la admisibilidad de la demanda y que estos asuntos deben, por tanto, ser determinados en forma separada antes de cualquier procedimiento sobre el fondo del caso. En la providencia se tiene buen cuidado de especificar una de dichas circunstancias, a saber, "el hecho de que, según los Estados Unidos, la Corte manifiestamente carece de competencia para conocer de la Demanda de Palestina".

- Segundo considerando: que es necesario que la CIJ esté informada de todos los fundamentos de hecho y de derecho en los que las partes se basan respecto de la cuestión de la competencia de la Corte y la admisibilidad de la demanda.

Primer Considerando: debate separado sobre competencia. En el considerando mencionado, la CIJ invoca lo dispuesto en el párrafo 2 del artículo 79 del Reglamento, el cual dispone: “2. No obstante lo dispuesto en el párrafo 1, una vez presentada la demanda y después de que el presidente se haya reunido y celebrado consultas con las partes, la Corte podrá decidir la necesidad de pronunciarse sobre cualquier cuestión de competencia y admisibilidad por separado". ${ }^{22}$

El artículo 79 del Reglamento de la Corte se titula “Excepciones Preliminares" y regula la manera como se impugna la competencia de la CIJ o la admisibilidad de la demanda. De ordinario, esto lo hace el Estado demandado después de que el demandante ha presentado su primer alegato escrito, que se deno-

22 En el párrafo 1 se fija el plazo para que el Estado que desee impugnar la competencia de la Corte o la admisibilidad de la demanda formule excepciones preliminares, el cual es de tres meses a partir de la presentación del primer alegato escrito de la otra parte sobre el fondo. 
mina "Memoria". ${ }^{23}$ Anteriormente, no había un plazo perentorio para hacer esto, siempre que se hiciera antes de que el demandado presentara su alegato de respuesta sobre el fondo, es decir, la contramemoria.

En la reforma al Reglamento del año 2000 se introdujo un plazo específico para plantear las excepciones que ahora es de tres meses a partir de la fecha de presentación de la Memoria. Esto significa que, después de que la CIJ ha fijado plazos para la presentación de la primera pieza del procedimiento escrito, que normalmente es una memoria por el demandante y la subsecuente contramemoria por el demandado, este último tiene la opción de, en lugar de presentar su contramemoria, interponer excepciones preliminares, con lo cual se suspende el procedimiento sobre el fondo. ${ }^{24}$

Pero la práctica de los Estados desde mediados de los años setenta desarrolló un mecanismo alternativo consistente en que en la primera reunión del presidente de la CIJ con los agentes de las partes el representante del demandado informaba que en opinión de su gobierno la CIJ no tenía competencia y, por tanto, debía examinar primero y en forma separada esta cuestión, antes de iniciar siquiera el procedimiento sobre el fondo. La idea subyacente es que, si las circunstancias del caso lo ameritan, la CIJ puede siempre decidir que el primer intercambio de piezas escritas del procedimiento-la memoria y la contramemoria- se concentren exclusivamente en las cuestiones de competencia o admisibilidad que hayan podido surgir, sin entrar en cuestiones relativas al fondo, las cuales únicamente habrá lugar a examinar si la CIJ se cerciora primero de que efectivamente posee competencia o de que la demanda es admisible. En estos casos, no cabe hablar de "excepciones preliminares" en sentido estricto, sino de un debate judicial previo o separado relativo a las cuestiones de competencia y admisibilidad. Tampoco hay lugar a la suspensión del procedimiento escrito sobre el fondo, ya que, en realidad, este no ha comenzado todavía.

En la reforma al Reglamento de la Corte, aprobada en el año 2000, se decidió codificar esta práctica e incorporarla en el texto del propio artículo 79, en calidad de un nuevo párrafo 2, a manera de excepción a la regla general del párrafo 1 sobre oportunidad para la formulación de las excepciones preliminares propiamente dichas. Las restantes reglas procesales contenidas en ese artículo, además, sobre contenido y formato de los alegatos y otras cuestiones relacionadas, se aplican por igual a los casos del párrafo 1 (excepciones preliminares stricto sensu) y a los del párrafo 2 (debate previo y separado sobre jurisdicción y admisibilidad).

23 Ver Juan José Quintana, “La impugnación de la competencia en asuntos contenciosos ante la Corte Internacional de Justicia", en Anuario Colombiano de Derecho Internacional 1 (1) (2008), p. 19. Ibid., pp. 19-20. 
En la práctica de la CIJ, este procedimiento alternativo fue utilizado también en varios casos de no comparecencia, es decir, cuando en una etapa muy temprana del procedimiento el Estado nombrado como demandado hizo saber a la Corte que en su opinión ella no tenía competencia o la demanda no era admisible y, por tanto, debía abstenerse de continuar con el procedimiento. En varios casos de este tipo la CIJ tomó ex officio la decisión de examinar primero y por separado las cuestiones de competencia y admisibilidad y, siguiendo la práctica usual en casos de no comparecencia, les ofreció a ambas partes, es decir, tanto al Estado demandante como al Estado que anunció que no comparecería, la oportunidad procesal de presentar alegatos escritos dedicados exclusivamente a esas cuestiones preliminares. ${ }^{25}$

En efecto, esto es lo que ha hecho la CIJ en el caso de Palestina v. Estados Unidos, con la única diferencia de que esta es apenas la segunda vez que esto sucede después de la reforma del Reglamento de $2000 .{ }^{26} \mathrm{Es}$, por tanto, una de las primeras ocasiones en la que el párrafo 2 del artículo 79 del Reglamento es aplicado en un contexto de no comparecencia del Estado demandado, y por esto la providencia que se comenta puede tener un interés especial para quienes siguen los desarrollos procesales en los casos ante la CIJ.

Desde el punto de vista del manejo de cuestiones de competencia y admisibilidad en casos de no comparecencia, también es interesante que la CIJ haya escogido seguir este procedimiento y hacer uso del párrafo 2 del artículo 79 del Reglamento, lo cual parecería señalar un derrotero para casos futuros. El último caso hasta ahora en el cual un Estado nombrado como demandado decidió no comparecer y surgieron cuestiones de competencia o admisibilidad fue el de los Rehenes de Estados Unidos en Teherán (Estados Unidos v. Irán), en el cual el demandado no compareció y manifestó por varios canales que la CIJ carecía de competencia, pero allí la Corte recurrió a un procedimiento diferente. ${ }^{27}$ Con base en la Providencia que se comenta, todo parecería indicar

25 Los siguientes son los casos de no comparecencia en los que la Corte decidió que examinaría las cuestiones de su jurisdicción o la admisibilidad de la demanda en forma separada y anterior al inicio del procedimiento sobre el fondo: i) Corte Internacional de Justicia, “Caso relativo a las Pesquerías - Jurisdicción (Reino Unido v. Islandia y Alemania v. Islandia)", Providencias de 18 de agosto de 1972, ICJ Reports 1972, p. 181, 188; ii) "Caso relativo a los Ensayos Nucleares (Australia v. Francia y Nueva Zelandia v. Francia)", Providencias de 22 de junio de 1973, ICJ Reports 1973, p. 99, 135; iii) "Caso relativo a los Prisioneros de Guerra Pakistaníes", Providencia de 13 de julio de 1973, ICJ Reports 1973, p. 330; iv) “Caso relativo a la Plataforma Continental del Mar Egeo", Providencia de 11 de septiembre de 1976, ICJ Reports 1976, p. 3.

26 Lo mismo ocurrió en el caso en curso sobre el Laudo Arbitral del 3 de octubre de 1899 (Guyana v. Venezuela), decisión en la cual la Corte establece que resolverá los asuntos de jurisdicción que se plantean con anterioridad al fondo del caso, e invita a Venezuela, Estado que ha manifestado que no comparecerá en el proceso, para que manifieste sus objeciones a la competencia de la Corte en un alegato formal (Corte Internacional de Justicia, "Caso relativo al Laudo Arbitral del 3 de octubre de 1899 (Guyana v. Venezuela)", Providencia de 19 de junio de 2018).

27 En efecto, en el caso de los Rehenes de los EE. UU. en Teherán, la Corte no solicitó alegatos separados sobre jurisdicción y admisibilidad, sino que simplemente fijó términos para el depósito de una Memoria y una Contramemoria. La Corte no explicó cuál esperaba que fuera el contenido de esos escritos, pero en su decisión sobre medidas provisionales, después de indicar que poseía competencia prima facie, incluyó la salvaguarda usual indicando que esta decisión no prejuzgaba sobre la cuestión de la competencia de la Corte, por lo que dejaba protegidos los derechos del gobierno de Irán para presentar argumentos contra su competencia o so- 
que el mecanismo previsto en el párrafo 2 del artículo 79 suministra ahora una valiosa herramienta para que la CIJ maneje las cuestiones de competencia y admisibilidad que suelen surgir en aquellos casos en los que el Estado nombrado como demandado toma la decisión de no comparecer en el proceso.

Segundo considerando: contenido de la primera ronda de alegatos. En el segundo de los considerandos mencionados, la CIJ recuerda que es necesario para ella estar informada de todos los fundamentos de hecho y de derecho en los que las partes se basan respecto de la cuestión de la competencia de la CIJ.

Se reafirma así, una vez más, que el procedimiento en curso tiene un ámbito material cuidadosamente delimitado, ya que los argumentos que la CIJ espera recibir de ambas partes deben referirse única y exclusivamente a la cuestión de la competencia de la Corte para conocer del caso y la admisibilidad de la demanda.

Secuencia de los alegatos: memoria por Palestina y contramemoria por Estados Unidos. Un último aspecto de la Providencia que conviene destacar es la decisión de la CIJ de que sea Palestina quien presente el primer alegato, es decir, la Memoria. El fundamento de esta decisión es claramente el párrafo 3 del artículo 79 del Reglamento (citado en los párrafos iniciales de la Providencia), norma que autoriza a la CIJ a fijar los plazos para "los alegatos relativos a la competencia y la admisibilidad [...] en el orden por ella establecido".

En primer lugar, como se señaló, en los casos de no comparecencia la CIJ es siempre muy escrupulosa en fijar plazos para la presentación de alegatos por ambas partes, con el fin de salvaguardar hasta donde sea posible el principio de igualdad de armas. Es por tanto enteramente normal que se haya fijado también un plazo para la Contramemoria de Estados Unidos, a pesar de que hasta ahora las autoridades de ese país han dejado entrever que no piensan participar en el procedimiento. En todo caso, al igual que en todos los restantes casos de no comparecencia, nada obsta para que en un futuro el Gobierno de ese país cambie de opinión y decida presentar su Contramemoria, o decida más adelante participar en las audiencias orales, si las circunstancias lo permiten. ${ }^{28}$

bre el fondo (Corte Internacional de Justicia, “Caso relativo a los Rehenes de los EE.UU. en Teherán (Estados Unidos v. Irán)", Providencia sobre protecciones provisionales de 15 de diciembre de 1979, ICJ Reports 1979, p. 20, pár. 45). Como había hecho en el procedimiento sobre medidas provisionales, la Corte prestó atención a las comunicaciones informales del Estado que no compareció relacionadas con asuntos de la competencia de la Corte. Sin embargo, lo hizo al considerar el fondo del caso, en una fase única que redujo notablemente la extensión de la totalidad del procedimiento. La Corte, por tanto, unió de forma automática los asuntos preliminares con los de fondo, sin permitir una fase previa dedicada exclusivamente a asuntos jurisdiccionales. Al respecto, se deben tener en cuenta los comunicados de prensa de la Corte de fecha 20 de marzo y 7 de abril
de 2020, en los que manifiesta que, dada la pandemia del covid-19, no convocará a audiencias en los casos en curso por un tiempo y hasta que la situación mejore. 
En segundo lugar, teniendo en cuenta la actitud de Estados Unidos, el primer alegato escrito por presentarse debía ser por fuerza una Memoria por Palestina, a pesar de que se desconocen las razones por las cuales aquel sostiene que la CIJ carece de competencia. Los asesores jurídicos de Palestina deben ahora hacer un esfuerzo muy grande de imaginación y anticipar hasta donde sea posible las eventuales objeciones que pueda tener Estados Unidos respecto del tema de la competencia de la CIJ o la admisibilidad de la demanda.

En los casos normales de impugnación de la competencia, como el Estado demandado es el que presenta estas objeciones bajo la forma de un escrito de Excepciones Preliminares, la carga del demandante es menor, ya que se limita a responder estas con unas observaciones escritas (art. 79, párr. 5 del Reglamento). Pero si no hay excepciones preliminares formales y el demandante en el caso principal es quien debe presentar el primer alegato, se ve forzado a elaborar este documento actuando un poco a oscuras, sobre la base de meras hipótesis de lo que podrían contener unas eventuales objeciones de jurisdicción o de admisibilidad que el demandado pueda sentirse justificado en plantear ante la CIJ. Todo esto resulta marcadamente artificial y genera dificultades prácticas para los abogados del demandante, pero ello es inevitable dada la forma como está planteado el litigio y el procedimiento que se ha debido seguir para agotar esta fase preliminar sobre competencia de la CIJ.

Estados Unidos como parte en el caso. Aunque Estados Unidos no ha designado un agente en el caso y tampoco envió un representante a la reunión del 5 de noviembre de 2018, no cabe duda de que, jurídicamente, tiene la condición de parte en el proceso.

En el régimen de la CIJ la condición de parte en un caso se adquiere por el mero hecho de que un Estado haya sido nombrado, como demandado en una demanda introductoria de instancia. ${ }^{29}$ Ahora bien, si la demanda misma no cumple el requisito de indicar un título concreto de competencia, es decir, uno o más instrumentos jurídicos como un tratado o una declaración unilateral en el que conste que el Estado contra el cual se dirige la demanda ha aceptado efectivamente la competencia de la CIJ, el Secretario no le da curso a la demanda y se limita a informarle del hecho al otro Estado, en lo que efectivamente constituye una invitación del demandante a que el caso sea sometido a la CIJ en forma voluntaria. Esto figura en el párrafo 5 del artículo 38 del Reglamento de la Corte y la práctica enseña que, aunque estas "invitaciones" han sido cursadas en numerosas ocasiones, solo en muy contadas de ellas el Estado nombrado como demandado potencial ha aceptado de buena gana acudir ante la CIJ y litigar el caso de que se trate.

29 Si el caso fue introducido mediante la notificación de un Acuerdo Especial o "compromis", la condición de parte en el caso se adquiere por todos los Estados que sean partes en ese instrumento desde el momento en que se produce su notificación a la Corte (Corte Internacional de Justicia, "Reglamento de la Corte", 14 de abril de 1978, art. 39). 
Pero si la demanda menciona un título de jurisdicción, por precario que este pueda parecer a primera vista, el Secretario está obligado a darle curso, es decir, a notificarla al Estado nombrado como demandado, informar al Secretario General de la ONU y a todos los Estados partes en el Estatuto y abrir un caso en la Lista General de la CIJ, asignándole un número de orden y un nombre oficial. Esto es lo que sucedió con la demanda presentada por Palestina el 28 de septiembre de 2018, ya que en ella se identificaba al Protocolo Facultativo de la Convención de Viena de 1961 como el instrumento por medio del cual -según Palestina- los dos países aceptaron la competencia de la CIJ en relación con esa controversia específica. El caso fue formalmente abierto y quedó inscrito en la Lista General bajo el número de orden 176, con el nombre oficial "Traslado de la Embajada de los Estados Unidos a Jerusalén (Palestina v. Estados Unidos)". Para todos los efectos legales, entonces, a partir del momento de la presentación de la demanda, Estados Unidos, como demandado, es parte en el caso en las mismas condiciones que Palestina, como demandante, y a pesar de que haya decidido no comparecer en el procedimiento o de que opte por no presentar una defensa, no pierde esa condición.

Las cuestiones ante la CIJ: acceso, competencia y admisibilidad. Como se dijo, según la Providencia que se comenta, lo que está ante la CIJ en estos momentos son "las cuestiones de la competencia de la Corte y la admisibilidad de la demanda". En el contexto del caso, quedan comprendidos bajo esta fórmula tres asuntos claramente diferenciados: i) el acceso de Palestina a la CIJ; ii) la competencia de la CIJ para ocuparse de la demanda; y iii) la admisibilidad de la demanda misma.

Acceso: en el primer párrafo de la parte preambular de la Providencia la CIJ registra la formulación por Palestina de una declaración el 4 de julio de 2018, mediante la cual ese Estado acepta la competencia de la Corte "para la solución de todas las controversias que puedan surgir o que hayan surgido y que estén cubiertas por el artículo I del Protocolo Facultativo de la Convención de Viena sobre Relaciones Diplomáticas relativo a la Solución Obligatoria de Controversias". Lo más revelador de este párrafo es que se menciona allí en forma explícita que esta declaración fue hecha "de conformidad con la Resolución 9 (1946) del Consejo de Seguridad del 15 de octubre de 1946, adoptada en virtud de las facultades conferidas al Consejo por el artículo 35, párrafo 2 del Estatuto de la Corte".

Está muy claro, entonces, que la CIJ deberá ocuparse en primerísimo lugar de si esta es una declaración válida, lo que implica antes que nada definir si Palestina estaba en capacidad de hacerla, es decir, si califica como "Estado" a la luz de dicha resolución $y$, por tanto, debe considerarse que posee acceso a la Corte bajo la regla especial del artículo 35 (2) del Estatuto. En la providencia se menciona que en una carta del 2 de noviembre de 2018 la Asesora Jurídica del Departamento de Estado le expresó a la CIJ ciertas ra- 
zones por las cuales esta carece de competencia en relación con la demanda presentada por Palestina, pero no se precisa si entre estas razones había alguna referida a la cuestión del acceso.

Habrá que esperar a ver si el asunto se plantea ante la CIJ en algún momento, pero, como Palestina es quien debe presentar el primer alegato sobre los temas de competencia, parecería lógico que sus representantes eviten cuidadosamente referirse a este asunto y simplemente den por sentado que, gracias a la declaración mencionada, Palestina posee acceso. En esas circunstancias, podría resultar conveniente que Estados Unidos informe a la CIJ sus puntos de vista respecto de este aspecto del caso, ya sea mediante un alegato formal o, si confirman su determinación de no comparecer en el caso, mediante un documento extraprocesal que hagan llegar a la CIJ por el canal diplomático.

Competencia: un aspecto interesante de la carta del Departamento de Estado a la CIJ es que en ella se dice que, dado que Estados Unidos no se considera legalmente vinculado con Palestina ni por la Convención ni por el Protocolo Facultativo sobre Solución de Controversias, en opinión de ese Gobierno "es manifiesto que la Corte carece de competencia con respecto a la Demanda" y que, por tanto, el caso debería removerse de la Lista General de la CIJ.

Aquí Estados Unidos le apuntó a un concepto que ha sido desarrollado en la jurisprudencia de la CIJ y que se denomina "ausencia manifiesta de competencia" (manifest lack of jurisdiction). En términos muy sencillos, si desde el comienzo mismo de un caso se hace patente para la CIJ que ella carece de competencia, el tribunal se siente autorizado para remover el caso de la Lista, incluso empleando para ello un procedimiento sumario (summary dismisal). ${ }^{30} \mathrm{~A}$ la inversa, si la Corte considera que en un caso que le ha sido sometido regularmente y en el cual el demandado le pide formalmente que suprima el caso de la Lista General, no se presenta la situación de "ausencia manifiesta de competencia", ella no se considera autorizada a acceder a dicha solicitud..$^{31}$ Esto tiene la implicación de que la CIJ únicamente podrá tomar una decisión definitiva sobre la cuestión de su competencia después de que se surta ante ella un procedimiento incidental relativo a ese asunto, de conformidad con lo dispuesto en el artículo 79 de su Reglamento.

Si se observa bien, esto es lo que sucedió en este caso ya que, al adoptar la providencia del 15 de noviembre de 2018 y organizar dicho procedimiento incidental, bajo los párrafos 2 y 3 de su Reglamento, la CIJ estaba efectiva-

30 Quintana, Litigation at the International Court of Justice, op. cit., pp. 481-485, 741-745.

31 Corte Internacional de Justicia, “Caso relativo a las Actividades Armadas II (República Democrática del Congo v. Ruanda)", Medidas Provisionales, Providencia de 10 de julio de 2002, ICJ Reports 2002, p. 249, pár. 91. 
mente rechazando la solicitud de Estados Unidos en el sentido de remover el caso de su Lista.

Admisibilidad: finalmente, también es interesante registrar que, al parecer, la comunicación del Departamento de Estado hacía referencia únicamente a la competencia de la CIJ para conocer de la demanda de Palestina y no a la admisibilidad de esta última, la cual es formal y materialmente diferente de la cuestión de competencia. Parecería, entonces, que fue la Corte misma la que añadió esa cuestión al decidir que la primera ronda de escritos se concentre en estos temas.

El comentario es pertinente porque en un caso en curso muy similar, en el cual la CIJ ha empleado un procedimiento virtualmente idéntico, la providencia adoptada por esta luego de la reunión del presidente con el agente del Estado compareciente (apenas unos meses antes de la decisión que se comenta en Palestina v. Estados Unidos) se menciona únicamente la cuestión de la competencia y se deja por fuera del todo la cuestión de la admisibilidad. ${ }^{32}$ No está del todo clara la razón de esta diferencia ni las implicaciones que puede tener a futuro.

\section{Consideraciones finales}

El litigio entre Palestina y Estados Unidos, surgido tras la relocalización de la embajada de Estados Unidos en Israel a la ciudad de Jerusalén le ofrece a la Corte Internacional de Justicia una buena oportunidad para avanzar su jurisprudencia sobre ciertas cuestiones relativas al procedimiento que se sigue ante ella.

Junto con la alta carga política de la controversia, que sin duda llamará la atención de cualquier ciudadano del globo, el caso en mención conlleva debates procedimentales y jurídicos de hondo calado que le permitirán a la CIJ pronunciarse, entre otros asuntos, sobre la capacidad legal de una entidad para iniciar un proceso contencioso ante ella contra otro Estado, es decir, si posee acceso a la Corte, lo cual está ligado a determinar si Palestina puede considerarse o no un Estado en derecho internacional, por lo menos para los efectos específicos de comparecer ante ese Tribunal.

A su turno, y si la respuesta a la pregunta anterior es afirmativa, la CIJ podría manifestar su posición jurídica sobre la cuestión subsidiaria de si a la luz de los instrumentos invocados por Palestina -la Convención y su Protocolo Facultativo sobre Solución de Controversias- ella posee competencia sobre la controversia. En ese proceso, la CIJ deberá analizar si las adhesiones de Pales-

32 Corte Internacional de Justicia, "Caso relativo al Laudo Arbitral del 3 de octubre de 1899 (Guyana v. Venezuela)", cit. 
tina a dichos tratados son válidas, $\mathrm{y}$, sobre todo, si resultan oponibles frente a Estados Unidos, en presencia de enérgicas objeciones expresadas oportunamente por este último. Igualmente, se anticipa que la CIJ tendrá ocasión de hacer referencia a la admisibilidad de la demanda de Palestina, una cuestión diferente del asunto de la competencia, y de mucho interés para los abogados internacionalistas que siguen los litigios ante la Corte.

Es posible, sin embargo, que la CIJ trate este asunto como una cuestión sumaria, y no resuelva todas las apasionantes inquietudes legales que genera el caso y que han dado lugar a la publicación de numerosos artículos de los seguidores de estas materias. En cierta medida, ello dependerá de si Estados Unidos comparecerá en el proceso, así sea solamente para cuestionar la competencia de la CIJ, y en qué términos lo hace, o de cómo aborde el asunto el equipo legal de Palestina. En todo caso, la oportunidad está servida para que la Corte se pronuncie sobre estos asuntos y avance, en consecuencia, el derecho de los tratados, el derecho internacional en materia de los requisitos para obtener la condición de Estado y el derecho procesal internacional en cuanto a los casos en curso ante ese tribunal.

\section{Bibliografía}

Kolb, Robert, The International Court of Justice, Oxford y Portland, Hart Publishing, 2013.

Quintana, Juan José, “La impugnación de la competencia en asuntos contenciosos ante la Corte Internacional de Justicia", en Anuario Colombiano de Derecho Internacional 1, 1 (2008), en https://revistas.urosario.edu.co/index. php/acdi/article/view/124.

Quintana, Juan José, Litigation at the International Court of Justice: Practice and Procedure, Leiden, Brill, 2015.

Rosenne, Shabtai, "Capacity to litigate in the International Court of Justice: Reflections on Yugoslavia in the Court", en British Yearbook of International Law 80, 1 (2009), pp. 217-243, DOI: https://doi.org/10.1093/bybil/80.1.217

Rosenne, Shabtai, The Law and Practice of the International Court, 1920-2015, Dordrecht, Brill Nijhoff, 2006.

Thirlway, Hugh, The International Court of Justice, London, Oxford University Press, 2016.

Vidmar, Jure, "Palestine v United States: Why the ICJ does not need to decide whether Palestine is a state", en European Journal of International Law (EJIL:Talk!) (2018), en https://www.ejiltalk.org/palestine-v-united-stateswhy-the-icj-does-not-need-to-decide-whether-palestine-is-a-state/. 
Whitman, Charles F., "Palestine's Statehood and Ability to Litigate in the International Court of Justice", en California Western International Law Journal 44, 1 (2013), en https://scholarlycommons.law.cwsl.edu/cwilj/vol44/iss1/4/

Zimmermann, Andreas, Christian Tomuschat y Karin Oellers-Frahm (eds.), The Statute of the International Court of Justice: A Commentary, London, Oxford University Press, 2006.

\section{Jurisprudencia}

Corte Internacional de Justicia, "Admisión a la ONU”, Opinión Consultiva de 28 de mayo de 1948, ICJ Reports 1948.

Corte Internacional de Justicia, "Caso Nottebohm (Liechtenstein v. Guatemala), Excepciones Preliminares", Sentencia de 18 de noviembre de 1953, ICJ Reports 1953.

Corte Internacional de Justicia, "Caso relativo al Derecho de Paso sobre Territorio Indio (Portugal v. India)", Excepciones Preliminares, Sentencia de 26 de noviembre de 1957, ICJ Reports 1957.

Corte Internacional de Justicia, “Caso relativo a las Pesquerías - Jurisdicción (Reino Unido v. Islandia y Alemania v. Islandia)", Providencias de 18 de agosto de 1972, ICJ Reports 1972.

Corte Internacional de Justicia, "Caso relativo a los Ensayos Nucleares (Australia v. Francia y Nueva Zelandia v. Francia)", Providencias de 22 de junio de 1973, ICJ Reports 1973.

Corte Internacional de Justicia, “Caso relativo a los Prisioneros de Guerra Pakistaníes”, Providencia de 13 de julio de 1973, ICJ Reports 1973.

Corte Internacional de Justicia, "Caso relativo a la Plataforma Continental del Mar Egeo", Providencia de 11 de septiembre de 1976, ICJ Reports 1976.

Corte Internacional de Justicia, "Caso relativo a los Rehenes de los EE. UU. en Teherán (Estados Unidos v. Irán)", Providencia sobre protecciones provisionales, 15 de diciembre de 1979, ICJ Reports 1979.

Corte Internacional de Justicia, "Caso relativo a la Aplicación de la Convención sobre la Prevención y el Castigo del Delito de Genocidio (Bosnia Herzegovina v. Serbia y Montenegro)", Excepciones Preliminares, Sentencia de 11 de julio de 1996, ICJ Reports 1996.

Corte Internacional de Justicia, “Caso relativo a la Legalidad del Uso de la Fuerza (Serbia y Montenegro v. Bélgica)", Medidas Provisionales, Providencia de 2 de junio de 1999, ICJ Reports 1999. 
Corte Internacional de Justicia, “Caso relativo a las Actividades Armadas II (República Democrática del Congo v. Ruanda)", Medidas Provisionales, Providencia de 10 de julio de 2002, ICJ Reports 2002.

Corte Internacional de Justicia, "Caso relativo a la Legalidad del Uso de la Fuerza (Serbia y Montenegro v. Francia)", Medidas Provisionales", Sentencia de 15 de diciembre de 2004, ICJ Reports 2004.

Corte Internacional de Justicia, "Caso relativo a la Aplicación de la Convención sobre la Prevención y el Castigo del Delito de Genocidio (Bosnia Herzegovina v. Serbia y Montenegro)", Fondo, Sentencia de 26 de febrero de 2007, ICJ Reports 2007.

Corte Internacional de Justicia, "Caso relativo a la Aplicación de la Convención sobre la Prevención y el Castigo del Delito de Genocidio (Croacia v. Serbia)", Excepciones Preliminares, Sentencia de 18 de noviembre de 2008, ICJ Reports 2008.

Corte Internacional de Justicia, "Caso relativo al Laudo Arbitral del 3 de octubre de 1899 (Guyana v. Venezuela)", Providencia de 19 de junio de 2018.

Corte Internacional de Justicia, "Caso relativo a la reubicación de la Embajada de los Estados Unidos a Jerusalén, (Palestina v. Estados Unidos)", Providencia de 15 de noviembre de 2018.

\section{Documentos legales}

Asamblea General de las Naciones Unidas, Resolución No. A/Res/67/19, 4 de diciembre de 2012.

Consejo de Seguridad de las Naciones Unidas, Resolución No. 9 (1946), 15 de octubre de 1946.

Corte Internacional de Justicia, "Reglamento de la Corte Internacional de Justicia", 14 de abril de 1978.

Corte Internacional de Justicia, Estatuto de la Corte Internacional de Justicia, 14 de abril de 1978.

Organización de las Naciones Unidas, "Carta de las Naciones Unidas", 26 de junio de 1945.

Secretaría General de las Naciones Unidas, Convención de Viena sobre Relaciones Diplomáticas de 1961, 2 de marzo - 18 de abril de 1961.

Secretaría General de las Naciones Unidas, Protocolo Facultativo sobre la Jurisdicción Obligatoria para la Solución de Controversias de la Convención de Viena sobre Relaciones Diplomáticas de 1961, 2 de marzo - 18 de abril de 1961. 